\title{
Intrinsic Charm Flavor and Helicity Content in the Proton*
}

\author{
Xiaotong Song \\ Institute of Nuclear and Particle Physics \\ Jesse W. Beams Laboratory of Physics \\ Department of Physics, University of Virginia, \\ Charlottesville, VA 22904, USA
}

\begin{abstract}
Contributions to the quark flavor and spin observables from the intrinsic charm in the proton are discussed in the SU(4) quark meson fluctuation model. Our results suggest that the probability of finding the intrinsic charm in the proton is less than $1 \%$. The intrinsic charm helicity is small and negative, $\Delta c \simeq-(0.003 \sim 0.015)$. The fraction of the total quark helicity carried by the intrinsic charm is less than $2 \%$, and $c_{\uparrow} / c_{\downarrow}=35 / 67$.

PACS numbers: 14.65.Dw, 12.39.Fe, 11.30.Hv, 14.20.Dh
\end{abstract}

\section{Introduction}

The intrinsic heavy quark component in the nucleon wave function has been suggested by many authors long time ago [1, 2]. This component, created from the quantum fluctuations associated with the bound state hadron dynamics, exists in the hadron over a long time independent of any external probe momentum. The probability of finding the intrinsic heavy quarks in the hadron is completely determined by nonperturbative mechanisms. On the other hand, the extrinsic heavy quarks are created on a short time scale in association with a large transverse momentum reaction and their distributions can be derived from QCD bremsstrahlung and pair production processes, which lead to standard QCD evolution. At the scale $m_{c}^{2}$ or lower, we only need to consider the intrinsic charm (IC) contribution. An interesting question is what will be the size of the IC contribution to the flavor and spin observables of the proton if the IC does exist. Since there is no direct experimental data of the IC content, one has to resort to the nucleon models (see e.g. [2, 3]) or combination of using the model and analysing the DIS data to obtain some information of the IC contribution (see e.g. 仙).

Although the SU(3) chiral quark model with symmetry breaking provides a useful nonperturbative tool in describing the quark spin, flavor [5] and orbital structure [6], the model is quite unnatural from the point of view of the standard model. According to the symmetric GIM model |7], one should deal with the weak axial current in the framework of SU(4) symmetry. It implies that the charm quark should be included in determining the spin, flavor and orbital structure of the nucleon. In an earlier report 8, the author has suggested an extended $\mathrm{SU}(4)$ version of the chiral quark model and presented some

\footnotetext{
*Electronic address: xs3e@virginia.edu;

* Invited paper to the 3rd Circum-Pan-Pacific Symposium on "High Energy Spin Physics", Beijing, October 8-13, 2001.;

** also Department of Physics, Zhejiang University, Hangzhou, Zhejiang, P. R. China
}

preliminary results. In the chiral quark model or more precisely the quark meson fluctuation model (some earlier works on this model see e.g. [9]), the nucleon structure is determined by its valence quark configuration and all possible quantum fluctuations of valence quarks into quarks plus mesons. In the $\mathrm{SU}(4)$ model, the charm or anti-charm quarks reside in the charmed mesons which are created by nonperturbative quantum quark-meson fluctuations. Hence these charm or anticharm quarks are essentially intrinsic.

\section{SU(4) model with symmetry breaking}

In the framework of SU(4) quark model, there are sixteen pseudoscalar mesons, a 15-plet and a singlet. In this paper, the contribution of the $\mathrm{SU}(4)$ singlet will be neglected. The effective Lagrangian describing interaction between quarks and the mesons is

$$
L_{I}=g_{15} \bar{q}\left(\begin{array}{cccc}
G_{u}^{0} & \pi^{+} & \sqrt{\epsilon} K^{+} & \sqrt{\epsilon_{c}} \bar{D}^{0} \\
\pi^{-} & G_{d}^{0} & \sqrt{\epsilon} K^{0} & \sqrt{\epsilon_{c}} D^{-} \\
\sqrt{\epsilon} K^{-} & \sqrt{\epsilon} K^{0} & G_{s}^{0} & \sqrt{\epsilon_{c}} D_{s}^{-} \\
\sqrt{\epsilon_{c}} D^{0} & \sqrt{\epsilon_{c}} D^{+} & \sqrt{\epsilon_{c}} D_{s}^{+} & G_{c}^{0}
\end{array}\right) q
$$

where $D^{+}=(c \bar{d}), D^{-}=(\bar{c} d), D^{0}=(c \bar{u}), \bar{D}^{0}=(\bar{c} u)$, $D_{s}^{+}=(c \bar{s})$, and $D_{s}^{-}=(\bar{c} s)$. The neutral charge components $G_{u(d)}^{0}$ and $G_{s, c}^{0}$ are defined as

$$
\begin{gathered}
G_{u(d)}^{0}=+(-) \frac{\pi^{0}}{\sqrt{2}}+\sqrt{\epsilon_{\eta}} \frac{\eta^{0}}{\sqrt{6}}+\zeta^{\prime} \frac{\eta^{\prime 0}}{4 \sqrt{3}}-\sqrt{\epsilon_{c}} \frac{\eta_{c}^{0}}{4} \\
G_{s}^{0}=-\sqrt{\epsilon_{\eta}} \frac{2 \eta^{0}}{\sqrt{6}}+\zeta^{\prime} \frac{\eta^{\prime 0}}{4 \sqrt{3}}-\sqrt{\epsilon_{c}} \frac{\eta_{c}^{0}}{4} \\
G_{c}^{0}=-\zeta^{\prime} \frac{3 \eta^{\prime 0}}{4 \sqrt{3}}+\sqrt{\epsilon_{c}} \frac{3 \eta_{c}^{0}}{4}
\end{gathered}
$$

with $\pi^{0}=(u \bar{u}-d \bar{d}) / \sqrt{2}, \eta^{0}=(u \bar{u}+d \bar{d}-2 s \bar{s}) / \sqrt{6}, \eta^{\prime 0}=$ $(u \bar{u}+d \bar{d}+s \bar{s}) / \sqrt{3}$, and $\eta_{c}^{0}=(c \bar{c})$. Similar to the $\mathrm{SU}(3)$ case, we define $a \equiv\left|g_{15}\right|^{2}$, which denotes the transition probability of splitting $u(d) \rightarrow d(u)+\pi^{+(-)}$. Hence $\epsilon a$, 
$\epsilon_{\eta} a$ and $\epsilon_{c} a$ denote the probabilities of splittings $u(d) \rightarrow$ $s+K^{-(0)}, u(d) \rightarrow u(d)+\eta^{(0)}$ and $u(d) \rightarrow c+\bar{D}^{0}\left(D^{-}\right)$ respectively. If the breaking effects are dominated by the mass differences, we expect $0<\epsilon_{c}<\epsilon \simeq \epsilon_{\eta}<1$.

In addition to the allowed fluctuations discussed in the $\mathrm{SU}(3)$ case, a valence quark ( $u$ or $d$ in the proton) is now allowed to split up or fluctuate to a recoil charm quark and a charmed meson. For example, a valence u-quark with spin-up, the allowed fluctuations are

$$
\begin{aligned}
& u_{\uparrow} \rightarrow d_{\downarrow}+\pi^{+}, \quad u_{\uparrow} \rightarrow s_{\downarrow}+K^{+}, \quad u_{\uparrow} \rightarrow u_{\downarrow}+G_{u}^{0}, \\
& u_{\uparrow} \rightarrow c_{\downarrow}+\bar{D}^{0} \\
& u_{\uparrow} \rightarrow u_{\uparrow} .
\end{aligned}
$$

Similarly, one can list the allowed fluctuations for $u_{\downarrow}$, $d_{\uparrow}, d_{\downarrow}, s_{\uparrow}$, and $s_{\downarrow}$. Similar to the $\mathrm{SU}(3)$ [6] case, the spin-up and spin-down quark or antiquark contents in the proton, up to first order of the quantum fluctuation, can be calculated.

\section{Quark flavor and spin contents}

We note that the quark flips its spin in the splitting processes $q_{\uparrow,(\downarrow)} \rightarrow q_{\downarrow,(\uparrow)}+$ meson, i.e. processes in (5) and (6), but not in $u_{\uparrow} \rightarrow u_{\uparrow}$. The quark helicity non-flip contributions in the splitting processes (5) and (6) are neglected, which is the basic assumption in the model.

\section{3.a. Flavor content in the nucleon}

The quark and antiquark flavor contents are

$$
\begin{gathered}
u=2+\bar{u}, \quad d=1+\bar{d}, \quad s=0+\bar{s}, \quad c=0+\bar{c} \\
\bar{u}=a\left[1+\tilde{A}^{2}+2(1-\tilde{A})^{2}\right], \quad \bar{d}=a\left[2\left(1+\tilde{A}^{2}\right)+(1-\tilde{A})^{2}\right] \\
\bar{s}=3 a\left[\epsilon+\tilde{B}^{2}\right], \quad \bar{c}=3 a\left[\epsilon_{c}+\tilde{D}^{2}\right],
\end{gathered}
$$

where $\tilde{A}, \tilde{B}, \tilde{C}$, and $\tilde{D}$ are defined similar to those in the $\mathrm{SU}(3)$ case. From (9), one obtains

$$
\bar{u} / \bar{d}=1-6 \tilde{A} /\left[(3 \tilde{A}-1)^{2}+8\right],
$$

and

$$
\bar{d}-\bar{u}=2 a \tilde{A}
$$

Similarly, one can obtain $2 \bar{c} /(\bar{u}+\bar{d}), 2 \bar{c} /(u+d), 2 \bar{c} / \sum(q+$ $\bar{q}$ ) and other flavor observables. One remark should be made here. Defining the ratio, $r \equiv \bar{u} / \bar{d}$, we obtain, from Eqs. (11) and (12),

$$
1 / 2 \leq \bar{u} / \bar{d} \leq 5 / 4
$$

which seems to be consistent with the experimental data shown in Table I.

\section{3.b. Helicity content in the nucleon}

Similarly, we obtain

$$
\Delta u=(4 / 3)\left[1-a\left(\epsilon+\epsilon_{c}+2 f\right)\right]-a, \quad \Delta c=-a \epsilon_{c},
$$

$$
\Delta d=(-1 / 3)\left[1-a\left(\epsilon+\epsilon_{c}+2 f\right)\right]-a, \quad \Delta s=-a \epsilon_{s},
$$

(where $f$ is generalization of $f_{S U(3)}$ defined in [5]) and

$$
\Delta \bar{q}=0, \quad(\bar{q}=\bar{u}, \bar{d}, \bar{s}, \bar{c}) .
$$

Several remarks are in order.

- In the splitting process $u_{\uparrow(\downarrow)} \rightarrow c_{\downarrow(\uparrow)}+\bar{D}^{0}$, the anticharm resides only in the charmed meson, e.g. $\bar{D}^{0}(\bar{c}, u)$. The probabilities of finding $\bar{c}_{\uparrow}$ and $\bar{c}_{\downarrow}$ are equal in the spinless charmed meson. Therefore $\Delta \bar{c}=0$. Similar discussion in the $\mathrm{SU}(3)$ case has led to $\Delta \bar{q}=0$ for $\bar{q}=\bar{u}, \bar{d}, \bar{s}$. The DIS data [10] seems to support this prediction.

- The charm quark helicity $\Delta c$ is nonzero as far as $\epsilon_{c}$ is nonzero. Analogous to the strange quark helicity, $\Delta c$ is definitely negative, because in the splitting processes, $u_{\uparrow(\downarrow)} \rightarrow c_{\downarrow(\uparrow)}+\bar{D}^{0}$ and $d_{\uparrow(\downarrow)} \rightarrow c_{\downarrow(\uparrow)}+D^{-}$, more $c_{\downarrow}$ is created than $c_{\uparrow}$, because of the probability of finding the valence $u$-quark in the zeroth approximation, $n_{p}^{(0)}\left(u_{\uparrow}\right)$, is dominant.

- From (10) and (14), using $\tilde{D}^{2}=\epsilon_{c} / 16$, one can see that the ratio

$$
\Delta c / \bar{c}=-16 / 51
$$

is a constant, which does not depend on any splitting parameters. This is a special prediction for the charm flavor in the $\mathrm{SU}(4)$ quark meson model. Combining (17) and (16), one obtains $c_{\uparrow} / c_{\downarrow}=$ $35 / 67$. For the strangeness, if $\zeta^{\prime}=0$, one has similar result, i.e. $\Delta s / \bar{s}=-3 / 10$ is also a constant and $s_{\uparrow} / s_{\downarrow}=7 / 13$.

\section{Numerical results and discussion.}

Since the effect arising from splitting (6) is smaller than those from (5), we expect the values of parameters $a$ and $\epsilon$ in $\mathrm{SU}(4)$ should be very close to those used in $\mathrm{SU}(3)$ version, where $a=0.145, \epsilon=0.46$. We choose $a=0.143$, $\epsilon=0.454$, and leave $\epsilon_{c}$ as a variable, then the quark flavor and helicity contents can be expressed as functions of $\epsilon_{c}$. To determine the value of $\epsilon_{c}$, we use the low energy hyperon $\beta$-decay data [11], $\Delta_{3}=1.2670 \pm 0.0035$. We find

$$
\epsilon_{c} \simeq 0.06 \pm 0.04
$$

Using only three parameters, $\left\{a, \epsilon, \epsilon_{c}\right\}$, the flavor and spin observables are calculated and listed in Table I and Table II respectively. For comparison, we also list the existing data and results given by $\mathrm{SU}(3)$ description and other models or analyses. One can see that the model satisfactorily describes almost all the existing data and also gives some new predictions. Several remarks are in order: 
- The theoretical uncertainties shown in the quantities in Tables I and II arise from the uncertainty of $\epsilon_{c}$ in (11). If the observable does not depend on $\epsilon_{c}$, such as $\bar{d}-\bar{u}, \bar{d} / \bar{u}, 2 \bar{s} /(\bar{u}+\bar{d})$, etc., there is no uncertainty for them. Two special quantities $\Delta c / \bar{c}$ and $\Delta s / \bar{s}$ are also independent of $\epsilon_{c}$ (see Table II).

- The $\mathrm{SU}(4)$ version predicts the IC component in the proton, $2 \bar{c} / \sum(q+\bar{q}) \simeq 1 \%$, which agrees with the predictions given in [2] and [4e], and is also close to the those given in [3a], [3d] and [4f]. We note that the IC component is almost one order of magnitude smaller than the intrinsic strange component $2 \bar{s} / \sum(q+\bar{q})$.

- Using similar approach given in a previous work (see Eq. (3.6) in [9c], we can show that $\langle 2 x \bar{c}(x)\rangle$ $/<\sum[x q(x)+x \bar{q}(x)]>$ is smaller than $2 \bar{c} / \sum(q+$ $\bar{q})$, where $q(\bar{q}) \equiv \int_{0}^{1} d x q(\bar{q})(x)$, and $<x q(\bar{q})(x)>\equiv$ $\int_{0}^{1} d x x q(\bar{q})(x)$. It implies that the fraction of the total quark momentum carried by the charm and anticharm quarks is less than $1 \%$.

- The prediction of intrinsic charm polarization, $\Delta c \simeq-0.009 \pm 0.006$ is close to the result $\Delta c=$ $-0.020 \pm 0.005$ given in the instanton $\mathrm{QCD}$ vacuum model [3c]. Our result is smaller than that given in $[3 \mathrm{~b}](\Delta c \simeq-0.3)$. However, the size of $\Delta c \simeq-5 \cdot 10^{-4}$ given in [3d] is even smaller. Hence further investigation in this quantity is needed.

- Taking $\epsilon_{c} \simeq 0.06$, one has $\Delta c / \Delta \Sigma \simeq-0.02$. This is consistent with the prediction given in [3c], but smaller than that given in [3b]. Combining with the fractions of the light quark helicities, we have $\Delta u / \Delta \Sigma \simeq 2.17, \Delta d / \Delta \Sigma \simeq-0.99, \Delta s / \Delta \Sigma \simeq$ -0.16 , and $\Delta c / \Delta \Sigma \simeq-0.02$. One can see that the $u$-quark helicity is positive (parallel to the nucleon spin) and about two times larger than the total quark helicity $\Delta \Sigma$. However, the $d-, s^{-}$, and $c$-helicities are all negative (antiparallel to the nucleon spin), and their sizes are decreased as

$$
\Delta d: \Delta s: \Delta c \simeq 1: 10^{-1}: 10^{-2} .
$$

Compare to the strange helicity $\Delta s$, the IC helicity is one order of magnitude smaller.

To summarize, we have discussed the IC contribution in the $\mathrm{SU}(4)$ quark meson model with symmetry breaking. Our results suggest that the probability of finding the IC in the proton is in the range $0.003 \sim 0.019$, and the IC helicity is small and negative, $\Delta c \simeq-(0.003 \sim 0.015)$. The fraction of the total quark helicity carried by the intrinsic charm is also small, $\Delta c / \Delta \Sigma \simeq-(0.007 \sim 0.035)$.

\section{Acknowledgments}

This work was supported in part by the U.S. DOE Grant, the Institute of Nuclear and Particle Physics, Department of Physics, University of Virginia, and the Commonwealth of Virginia.
[1] S. J. Brodsky, P. Hoyer, C. Peterson and N. Sakai, Phys. Lett. B93, 451 (1980); S. J. Brodsky, and C. Peterson, Phys. Rev. D23, 2745 (1980).

[2] J. F. Donoghue and E. Golowich, Phys. Rev. D15, 3421 (1977); H. He, X. Zhang, and Y. Zhao, High Energy Phys. Nucl. Phys. 7, 626 (1983).

[3] a) T. Hatsuda, and T. Kunihiro, Phys. Rep. 247, 221 (1994); b) A. Blotz and E. Shuryak, Phys. Lett. B439, 415 (1998); c) F. Araki, M. Musakhanov and H. Toki, hep-ph/9808290; d) M. V. Polyakov, A. Schafer, and O. V. Teryaev, Phys. Rev. D60, 051502 (1999); M. Franz, M. V. Polyakov, and K. Goeke, Phys. Rev. D62, 074024 (2000).

[4] a) E. Hoffmann and R. Moore, Z. Phys. C20, 71 (1983); b) B. W. Harris, J. Smith and R. Vogt, Nucl. Phys. B461, 181 (1996); c) R. Vogt and S. J. Brodsky, Nucl. Phys. B478, 311 (1996); d) G. Ingelman and M. Thunman, Z. Phys. C73, 505 (1997); e) Y. A. Golubkov, Phys. Atom. Nucl. 63, 606 (2000); f) S. J. Brodsky, and I. Schmidt, Phys. Rev. D43, 179 (1991); g) J. Blumlein and W. L. van Neerven, Phys. Lett. B450, 417 (1999).

[5] X. Song, Phys. Rev. D57, 4114 (1998).

[6] X. Song, Int. J. Mod. Phys. A16, 2673 (2001).
[7] S. L. Glashow et al., Phys. Rev. D2, 2185 (1970).

[8] X. Song, Internal Report, ICTP, June (1998).

[9] a) E. J. Eichten, I. Hinchliffe and C. Quigg, Phys. Rev. D45, 2269 (1992); Phys. Rev. D47, 747 (1993); b) T. P. Cheng and L.-F. Li, Phys. Rev. Lett. 74, 2872 (1995); c) X. Song, J. S. McCarthy and H. J. Weber, Phys. Rev. D55, 2624 (1997).

[10] K. Abe, et al., Phys. Rev. Lett. 79, 26 (1997).

[11] Particle Data Group, D. E. Groom, et al., Eur. Phys. J. C15, 1 (2000).

[12] J. C. Peng, et al., E866/NuSea Collaboration, Phys. Rev. D58, 092004 (1998).

[13] P. Amaudruz et al., Phys. Rev. Lett. 66, 2712 (1991); M. Arneodo et al., Phys. Rev. D50, R1, (1994).

[14] A. Baldit, et al., NA51 Collaboration, Phys. Lett. B332, 244 (1994).

[15] A. O. Bazarko, et al., Z. Phys. C65, 189 (1995).

[16] J. Gasser, H. Leutwyler and M. E. Saino, Phys. Lett. B253, 252 (1991).

[17] S. J. Dong, J. F. Lagae and K. F. Liu, Phys. Rev. D54, 5496 (1996).

[18] P. Adams, et al., Phys. Rev. D56, 5330 (1997).

[19] B. Adeva, et al., Phys. Lett. B369, 93 (1996). 
Tables

TABLE I: Quark Flavor Observables.

\begin{tabular}{|c|c|c|c|}
\hline Quantity & Data & SU(4) [This paper] & $\mathrm{SU}(3)[6$ \\
\hline$\overline{\bar{d}-\bar{u}}$ & $\begin{array}{l}0.110 \pm 0.018 \\
0.147 \pm 0.039\end{array}$ & 0.111 & 0.143 \\
\hline $\bar{u} / \bar{d}$ & $\begin{array}{c}{[\bar{u}(x) / \bar{d}(x)]_{0.1<x<0.2}=0.67 \pm 0.06[12]} \\
{[\bar{u}(x) / \bar{d}(x)]_{x=0.18}=0.51 \pm 0.06[14]}\end{array}$ & 0.71 & 0.64 \\
\hline $2 \bar{s} /(\bar{u}+\bar{d})$ & $<2 x \bar{s}(x)>/<x(\bar{u}(x)+\bar{d}(x))>=0.477 \pm 0.051[15]$ & 0.66 & 0.76 \\
\hline $2 \bar{c} /(\bar{u}+\bar{d})$ & - & $0.083 \pm 0.055$ & 0 \\
\hline $2 \bar{s} /(u+d)$ & $<2 x \bar{s}(x)>/<x(u(x)+d(x))>=0.099 \pm 0.009[15]$ & 0.118 & 0.136 \\
\hline $2 \bar{c} /(u+d)$ & - & $0.015 \pm 0.010$ & 0 \\
\hline$(s+\bar{s}) / \sum(q+\bar{q})$ & $\begin{array}{c}<2 x \bar{s}(x)>/ \sum<x(q(x)+\bar{q}(x))>=0.076 \pm 0.022[15 \\
0.10 \pm 0.06[16] \\
0.15 \pm 0.03[17]\end{array}$ & $0.090 \pm 0.001$ & 0.103 \\
\hline$(c+\bar{c}) / \sum(q+\bar{q})$ & $\begin{array}{c}0.03[4 \mathrm{f}]^{*} \\
0.02[2]^{*} \\
0.01[4 \mathrm{e}]^{*} \\
0.005[3 \mathrm{a}, 3 \mathrm{~d}]^{*}\end{array}$ & $0.011 \pm 0.008$ & 0 \\
\hline$\sum \bar{q} / \sum q$ & $\sum<x \bar{q}(x)>/ \sum<x q(x)>=0.245 \pm 0.005[15]$ & $0.230 \pm 0.004$ & 0.231 \\
\hline
\end{tabular}

TABLE II: Quark Spin Observables

\begin{tabular}{|c|c|c|c|}
\hline \hline Quantity & Data & $\mathrm{SU}(4)[$ This paper] & $\mathrm{SU}(3)[6]$ \\
\hline$\Delta u$ & $0.85 \pm 0.04[18]$ & $0.871 \pm 0.009$ & 0.863 \\
$\Delta d$ & $-0.41 \pm 0.04[18]$ & $-0.397 \pm 0.002$ & -0.397 \\
$\Delta s$ & $-0.07 \pm 0.04[[18]$ & $-0.065 \pm 0.000$ & -0.067 \\
\hline$\Delta c$ & $-0.020 \pm 0.004[3 \mathrm{c}]^{*}$ & $-0.009 \pm 0.006$ & 0 \\
& $-0.3[3 \mathrm{~b}]^{*}$ & & \\
& $-5 \cdot 10^{-4}[3 \mathrm{~d}]^{*}$ & & 0.200 \\
\hline$\Delta \Sigma / 2$ & $0.19 \pm 0.06[18]$ & $0.200 \pm 0.006$ & 0 \\
\hline$\Delta \bar{u}, \Delta \bar{d}$ & $-0.02 \pm 0.11[19]$ & 0 & 0 \\
\hline$\Delta \bar{s}, \Delta \bar{c}$ & - & 0 & 0 \\
\hline$\Delta c / \Delta \Sigma$ & $-0.08 \pm 0.01[3 \mathrm{~b}]^{*}$ & $-0.021 \pm 0.014$ & \\
\hline$\Delta s / \bar{s}$ & $-0.033[3 \mathrm{c}]^{*}$ & & -0.269 \\
\hline$\Delta c / \bar{c}$ & - & $-3 / 10$ & - \\
\hline$c_{\uparrow} / c_{\downarrow}$ & - & $-16 / 51$ & - \\
\hline$s_{\uparrow} / s_{\downarrow}$ & - & $35 / 67$ & 0.58 \\
\hline$\Gamma_{1}^{p}$ & $0.136 \pm 0.016[18]$ & $0.143 \pm 0.002$ & 0.142 \\
$\Gamma_{1}^{n}$ & $-0.041 \pm 0.007[10]$ & $-0.042 \pm 0.001$ & -0.042 \\
\hline$\Delta_{3}$ & $1.2670 \pm 0.0035[11]$ & $1.268 \pm 0.010$ & 1.260 \\
\hline$\Delta_{8}$ & $0.579 \pm 0.025[11]$ & $0.605 \pm 0.006$ & 0.600 \\
\hline
\end{tabular}

